\title{
CORRECTION
}

\section{Correction to: Safety and Effectiveness of Ipragliflozin in Elderly Versus Non-elderly Japanese Patients with Type 2 Diabetes: Subgroup Analysis of STELLA- LONG TERM}

Ichiro Nakamura $\cdot$ Hiroshi Maegawa $\cdot$ Kazuyuki Tobe $\cdot$

Satoshi Uno

Published online: May 20, 2021

(C) The Author(s) 2021

Correction to: Diabetes Ther (2021) 12:1359-1378

https://doi.org/10.1007/s13300-021-01042-w

In the original article, Table 2 was published with an error. The correct Table 2 is given below.

\section{Original}

Table 2 Adverse drug reactions in the safety analysis population (first age category analysis) of STELLA-LONG TERM, in pre-approval clinical trials, and STELLA-ELDER

The original article can be found online at https://doi. org/10.1007/s13300-021-01042-w.

I. Nakamura ( $\square)$

Operational Excellence, Medical Affairs Japan,

Astellas Pharma Inc., Tokyo, Japan

e-mail: ichiro.nakamura@astellas.com

H. Maegawa

Department of Medicine, Shiga University of

Medical Science, Shiga, Japan

K. Tobe

First Department of Internal Medicine, Graduate

School of Medicine and Pharmaceutical Sciences for

Research, University of Toyama, Toyama, Japan

S. Uno

Data Science, Development, Astellas Pharma Inc.,

Tokyo, Japan 


\begin{tabular}{|c|c|c|c|c|c|c|}
\hline \multirow[t]{2}{*}{ ADRs, $n(\%)$} & \multirow{2}{*}{$\begin{array}{l}\text { Pre-approval } \\
(n=1669)[2-7]\end{array}$} & \multicolumn{4}{|c|}{ STELLA-LONG TERM } & \multirow{2}{*}{$\begin{array}{l}\text { STELLA-ELDER } \\
(n=8505)[9]\end{array}$} \\
\hline & & $\begin{array}{l}\text { All } \\
(n=11,051)\end{array}$ & $\begin{array}{l}<65 \text { years } \\
(n=7894)\end{array}$ & $\begin{array}{l}\geq 65 \text { years } \\
(n=3157)\end{array}$ & $P$-value ${ }^{\text {a }}$ & \\
\hline Any ADR & $549(32.89)$ & $2129(19.27)$ & $1528(19.36)$ & $601(19.04)$ & 0.701 & $1438(16.9)$ \\
\hline Serious ADR & $14(0.8)$ & $210(1.90)$ & $122(1.55)$ & $88(2.79)$ & $<0.001$ & $127(1.5)$ \\
\hline \multicolumn{7}{|l|}{$\begin{array}{l}\text { ADRs of special } \\
\text { interest }\end{array}$} \\
\hline $\begin{array}{l}\text { Polyuria/ } \\
\text { pollakiuria }\end{array}$ & $168(10.0)$ & $612(5.54)$ & $471(5.97)$ & $141(4.47)$ & 0.002 & $170(2.0)$ \\
\hline $\begin{array}{l}\text { Volume } \\
\text { depletion }\end{array}$ & $73(4.5)$ & $243(2.20)$ & $167(2.12)$ & $76(2.41)$ & 0.345 & $266(3.1)$ \\
\hline $\begin{array}{l}\text { Skin } \\
\text { complications }\end{array}$ & $59(4.0)$ & $198(1.79)$ & $128(1.62)$ & $70(2.22)$ & 0.033 & $269(3.2)$ \\
\hline Renal disorder & $76(4.8)$ & $191(1.73)$ & $119(1.51)$ & $72(2.28)$ & 0.005 & $118(1.4)$ \\
\hline $\begin{array}{l}\text { Urinary tract } \\
\text { infection }\end{array}$ & $29(1.8)$ & $170(1.54)$ & $115(1.46)$ & $55(1.74)$ & 0.271 & $118(1.4)$ \\
\hline $\begin{array}{l}\text { Genital } \\
\text { infection }\end{array}$ & $32(2.0)$ & $161(1.46)$ & $126(1.60)$ & $35(1.11)$ & 0.053 & $166(2.0)$ \\
\hline Hepatic disorder & $17(1.0)$ & $133(1.20)$ & $110(1.39)$ & $23(0.73)$ & 0.004 & $19(0.2)$ \\
\hline $\begin{array}{l}\text { Cardiovascular } \\
\text { disease }\end{array}$ & $16(1.0)$ & $67(0.61)$ & $50(0.63)$ & $17(0.54)$ & 0.562 & $24(0.3)$ \\
\hline Hypoglycemia & $22(1.4)$ & $57(0.52)$ & $34(0.43)$ & $23(0.73)$ & 0.048 & $58(0.7)$ \\
\hline $\begin{array}{l}\text { Malignant } \\
\text { tumor }\end{array}$ & $4(0.2)$ & $51(0.46)$ & $19(0.24)$ & $32(1.01)$ & $<0.001$ & $11(0.1)$ \\
\hline $\begin{array}{l}\text { Cerebrovascular } \\
\text { disease }\end{array}$ & $4(0.2)$ & $48(0.43)$ & $29(0.37)$ & $19(0.60)$ & 0.090 & $36(0.4)$ \\
\hline $\begin{array}{l}\text { Ketone body } \\
\text { related events }\end{array}$ & $11(1.0)$ & $7(0.06)$ & $6(0.08)$ & $1(0.03)$ & $-{ }^{\mathrm{b}}$ & $2(0.02)$ \\
\hline Fracture & 0 & $4(0.04)$ & $4(0.05)$ & 0 & $-{ }^{\mathrm{b}}$ & $2(0.02)$ \\
\hline $\begin{array}{l}\text { Lower limb } \\
\text { amputation }\end{array}$ & 0 & 0 & 0 & 0 & $-{ }^{\mathrm{b}}$ & 0 \\
\hline
\end{tabular}

No. of patients (\%) are shown

ADR adverse drug reaction

${ }^{a}$ Chi-squared test for difference between BMI subgroups

${ }^{b}$ No $P$ value was calculated when at least one element of the contingency table was $<10$ 


\section{Correct Table 2}

Table 2 Adverse drug reactions in the safety analysis population (first age category analysis) of STELLA-LONG TERM, in pre-approval clinical trials, and STELLA-ELDER

\begin{tabular}{|c|c|c|c|c|c|c|}
\hline \multirow[t]{2}{*}{ ADRs, $n(\%)$} & \multirow{2}{*}{$\begin{array}{l}\text { Pre-approval } \\
(n=1669)[2-7]\end{array}$} & \multicolumn{4}{|c|}{ STELLA-LONG TERM } & \multirow{2}{*}{$\begin{array}{l}\text { STELLA-ELDER } \\
(n=8505)[9]\end{array}$} \\
\hline & & $\begin{array}{l}\text { All } \\
(n=11,051)\end{array}$ & $\begin{array}{l}<65 \text { years } \\
(n=7894)\end{array}$ & $\begin{array}{l}\geq 65 \text { years } \\
(n=3157)\end{array}$ & $P$-value ${ }^{\text {a }}$ & \\
\hline Any ADR & $549(32.89)$ & $2129(19.27)$ & $1528(19.36)$ & $601(19.04)$ & 0.701 & $1438(16.9)$ \\
\hline Serious ADR & $14(0.8)$ & $210(1.90)$ & $122(1.55)$ & $88(2.79)$ & $<0.001$ & $127(1.5)$ \\
\hline \multicolumn{7}{|l|}{$\begin{array}{l}\text { ADRs of special } \\
\text { interest }\end{array}$} \\
\hline $\begin{array}{l}\text { Polyuria/ } \\
\text { pollakiuria }\end{array}$ & $163(10.0)$ & $612(5.54)$ & $471(5.97)$ & $141(4.47)$ & 0.002 & $170(2.0)$ \\
\hline $\begin{array}{l}\text { Volume } \\
\text { depletion }\end{array}$ & $73(4.5)$ & $243(2.20)$ & $167(2.12)$ & $76(2.41)$ & 0.345 & $266(3.1)$ \\
\hline $\begin{array}{l}\text { Skin } \\
\text { complications }\end{array}$ & $59(3.5)$ & $198(1.79)$ & $128(1.62)$ & $70(2.22)$ & 0.033 & $269(3.2)$ \\
\hline Renal disorder & $76(4.6)$ & $191(1.73)$ & $119(1.51)$ & $72(2.28)$ & 0.005 & $118(1.4)$ \\
\hline $\begin{array}{l}\text { Urinary tract } \\
\text { infection }\end{array}$ & $29(1.8)$ & $170(1.54)$ & $115(1.46)$ & $55(1.74)$ & 0.271 & $118(1.4)$ \\
\hline $\begin{array}{l}\text { Genital } \\
\text { infection }\end{array}$ & $32(2.0)$ & $161(1.46)$ & $126(1.60)$ & $35(1.11)$ & 0.053 & $166(2.0)$ \\
\hline Hepatic disorder & $17(1.0)$ & $133(1.20)$ & $110(1.39)$ & $23(0.73)$ & 0.004 & $19(0.2)$ \\
\hline $\begin{array}{l}\text { Cardiovascular } \\
\text { disease }\end{array}$ & $16(1.0)$ & $67(0.61)$ & $50(0.63)$ & $17(0.54)$ & 0.562 & $24(0.3)$ \\
\hline Hypoglycemia & $22(1.4)$ & $57(0.52)$ & $34(0.43)$ & $23(0.73)$ & 0.048 & $58(0.7)$ \\
\hline $\begin{array}{l}\text { Malignant } \\
\text { tumor }\end{array}$ & $4(0.2)$ & $51(0.46)$ & $19(0.24)$ & $32(1.01)$ & $<0.001$ & $11(0.1)$ \\
\hline $\begin{array}{l}\text { Cerebrovascular } \\
\text { disease }\end{array}$ & $4(0.2)$ & $48(0.43)$ & $29(0.37)$ & $19(0.60)$ & 0.090 & $36(0.4)$ \\
\hline $\begin{array}{l}\text { Ketone body } \\
\text { related events }\end{array}$ & $11(0.7)$ & $7(0.06)$ & $6(0.08)$ & $1(0.03)$ & $-{ }^{b}$ & $2(0.02)$ \\
\hline Fracture & 0 & $4(0.04)$ & $4(0.05)$ & 0 & $-{ }^{b}$ & $2(0.02)$ \\
\hline $\begin{array}{l}\text { Lower limb } \\
\text { amputation }\end{array}$ & 0 & 0 & 0 & 0 & $-^{b}$ & 0 \\
\hline
\end{tabular}

No. of patients (\%) are shown

$A D R$ adverse drug reaction

a Chi-squared test for difference between BMI subgroups

b No $P$ value was calculated when at least one element of the contingency table was $<10$ 
Open Access. This article is licensed under a Creative Commons Attribution-NonCommercial 4.0 International License, which permits any non-commercial use, sharing, adaptation, distribution and reproduction in any medium or format, as long as you give appropriate credit to the original author(s) and the source, provide a link to the Creative Commons licence, and indicate if changes were made. The images or other third party material in this article are included in the article's Creative Commons licence, unless indicated otherwise in a credit line to the material. If material is not included in the article's Creative Commons licence and your intended use is not permitted by statutory regulation or exceeds the permitted use, you will need to obtain permission directly from the copyright holder. To view a copy of this licence, visit http://creativecommons.org/licenses/by$\mathrm{nc} / 4.0 /$. 